\title{
TURISMO Y COVID-19: UNA DISYUNTIVA SISTÉMICA
}

\author{
TOURISM AND COVID-19: A SYSTEMIC DILEMMA
}

\author{
Raúl Travé Molero \\ EAE Business School \\ rtrave@,ostelea.com \\ https://orcid.org/0000-0002-0032-1133 \\ Diego Santos González \\ EAE Business School \\ dsantos@ostelea.com \\ https://orcid.org/x
}

Recibido/Received: 17/12/2021

Modificado/Modified: 20/12/2021

Aceptado/Accepted: 22/12/2021

\section{RESUMEN}

La pandemia de COVID-19 forzó al sector turístico a detener una maquinaria mundial que llegó a mover a 1.460 millones de viajeros internacionales durante 2019 y generar 1,7 billones de dólares en exportaciones. El turismo, que se había convertido en tabla salvadora para muchas economías golpeadas por el desfalco de 2007, ha sufrido especialmente las consecuencias socio-económicas de la pandemia, pero al mismo tiempo se ha convertido en un terreno de innovación social en el que se han podido estudiar sincrónicamente fenómenos de adaptación que probablemente cambien de forma duradera las formas en las que practicamos y nos relacionamos, en diferentes niveles, con el turismo.

El presente texto sirve como presentación al monográfico "Turismo post-COVID-19 desde las Ciencias Sociales" que Barataria nos ha facilitado coordinar y como reflexión teórica sobre cómo la pandemia ha impactado en el turismo como hecho social total. La coincidencia generalizada, en las contribuciones a este número, de determinadas conclusiones es un buen indicador de la fiabilidad y relevancia del mismo.

\section{PALABRAS CLAVE}

Turismo; Ciencias Sociales; COVID-19, Cambio socio-cultural; Nuevas tendencias en turismo

\section{SUMARIO}

1. Introducción: La pandemia y el turismo. 2. Parón y nuevas tendencias. 3. Una disyuntiva sistémica. 4. El turismo post COVID-19

\begin{abstract}
The COVID-19 pandemic forced the tourism sector to stop a global machine that moved 1.46 billion international travellers during 2019 and generated 1.7 trillion dollars in exports. Tourism, which had become a lifeline for many economies hit by the 2008 previous economic crisis, has suffered especially the socio-economic consequences of the pandemic, but at the same time it has become a field of social innovation in which adaptation phenomena have been studied synchronously. These changes will probably change permanently the ways in which we practice and interact, at different levels, with tourism. This article serves at the same time as a presentation of the monographic "Post-COVID-19 Tourism from the Social Sciences" that Barataria has facilitated us to coordinate and as a theoretical reflection on how
\end{abstract}


the pandemic has impacted tourism as a total social fact. The general agreement, in the contributions to this number, of certain conclusions is a good indicator of its reliability and relevance.

\section{KEYWORDS}

Tourism; Social Science; COVID-19; Socio-cultural change; New trends in tourism

\section{CONTENTS}

1. Introduction: Pandemic and tourism. 2. Halt and new trends. 3. A systemic dilemma. 4. Post COVID19 tourism

\section{INTRODUCCIÓN: LA PANDEMIA Y EL TURISMO}

Cuando hace algo más de un año empezamos a dar forma a este monográfico sobre turismo post-COVID-19 lo hacíamos atenazados por la contradicción entre la necesidad de comprender qué estaba pasando, cómo estaba cambiando el turismo como fenómeno sociocultural, y las limitaciones propias de unas ciencias sociales que necesitan tiempo para recoger, analizar, comparar y comprobar datos. No obstante, la excepcionalidad del momento que vivimos hace que merezca la pena correr el riesgo de aventurarnos en análisis urgentes. Urgencia que, en el caso de los textos que aquí presentamos, no está reñida con el compromiso científico.

Casi dos años después de que la OMS declarase la COVID-19 como una pandemia, nuestras vidas siguen intensamente marcadas por las arremetidas de las diferentes olas, el muy desigual acceso a las vacunas dependiendo del PIB de cada país, las restricciones parciales de movimientos y actividades y los movimientos de protesta que ponen en cuestión desde las decisiones políticas de los gobiernos hasta la misma existencia del coronavirus. Llegando incluso a denunciar conspiraciones mundiales tras las vacunas (una conspiración que, por otro lado, estaría dejando de lado a casi tres cuartas partes de la humanidad). Quizá lo que entonces planteamos como una prospección de cambios sociales para después de la pandemia, ahora sea más bien una lectura de las formas que ha adoptado el turismo como fenómeno social en su convivencia -tal vez más larga de lo esperado- con los elementos arriba enumerados.

En este sentido, las autoras y autores que participan en este número han tenido que diseñar sus investigaciones, recoger datos cuantitativos y cualitativos, procesarlos y analizarlos en un contexto cambiante y volátil. Aun contra esto proporcionan una información muy valiosa. La coincidencia de determinadas conclusiones, alcanzadas de forma autónoma en contextos de estudio muy diferentes, es un buen indicador de la fiabilidad de estas investigaciones y de la relevancia de este monográfico: los cambios en la demanda, del turismo internacional al doméstico y de cercanía; la valorización de los destinos rurales y de naturaleza, identificados como más seguros; o la emergencia entre capas de la población cada vez más amplias de perspectivas críticas con los modelos turísticos más depredadores, pero también más extendidos y practicados. Esto último está ocurriendo de manera especialmente marcada en aquellos contextos donde el turismo se había convertido en un monocultivo. Lugares en los que tanto los impactos negativos previos a la pandemia como las consecuencias de esta han golpeado con más fuerza, especialmente a las capas más vulnerables de la población. Entre quienes, por cierto, se encuentran un buen número de trabajadoras y trabajadores del sector turístico. 
Algunos de estos cambios socio-culturales podrían atenuarse después de un tiempo cuando, como señala una de las contribuciones a este número "no nos acordemos de la pandemia". Sin duda veremos poderosos esfuerzos en esa dirección (por parte de las grandes empresas de la industria, pero también de las instituciones políticas en los destinos, de la OMT e incluso de la academia [Butcher, 2020]). No obstante, otros cambios pueden asentarse lo suficiente como para permanecer largo tiempo entre nosotros y quizá plantar una interesante batalla a modelos que ya han demostrado sus límites e impactos.

\section{PARÓN Y NUEVAS TENDENCIAS}

Desde el momento en que los países centrales del sistema-mundo capitalista empezaron a ser conscientes del alcance de la pandemia y fueron tomando medidas para contenerla e intentar controlar los impactos sobre sus sistemas de salud, la movilidad turística se detuvo casi por completo. Tan pronto como el 20 de abril de 2020 el 100\% de los países habían implantado restricciones a la movilidad, 97 habían cerrado total o parcialmente sus fronteras a los turistas y 65 habían suspendido total o parcialmente los vuelos internacionales (OMT, 2020: 6). Recordemos que, como industria, estamos hablando del tercer sector exportador legal a nivel mundial, por delante de la alimentación y la automoción. Así, según la OMT, el turismo movió más de 1400 millones de viajeros en 2018 y 2019 y generó cada uno de esos años alrededor de 1,7 billones de dólares como exportaciones en turismo internacional (OMT, 2019: 2), de los que 1,5 billones corresponderían a ingresos en los destinos (OMT, 2021: 4). Estas cifras corresponden, siempre según la OMT, al 7\% de las exportaciones mundiales (2021: 4).

Solo durante 2020 los flujos turísticos internacionales se habrían reducido en hasta un $75 \%$ dejando de mover alrededor de 1,2 billones de dólares en exportaciones (OMT, 2020: 10). Sin embargo, este parón también permitió que los residentes recuperasen lugares de juego y encuentro cedidos antes al turismo (y poco después, en bastantes casos, a las terrazas de la hostelería) o que se relajasen los precios de los alquileres gracias al regreso al mercado residencial de muchas viviendas transformadas en alojamientos turísticos. Según el INE, entre agosto de 2020 (ya en plena pandemia) y febrero de 2021 el número de viviendas de uso turístico pasó en España de 321.000 a 294.000, un 8,4\% menos (INE, 2020) No obstante, algunos estudios mostraban en el verano de 2021 que la mayoría de los propietarios que habían vuelto al alquiler residencial tenían intención de abandonarlo de nuevo por el turístico en cuanto "mejorase la situación" (Fotocasa, 2021). Este hecho viene a demostrar el fuerte impacto que el crecimiento de la demanda turística -causa y efecto al mismo tiempo de la implantación de ciertas plataformas digitales-, tiene sobre las condiciones de vida, especialmente de la población más vulnerable, en determinadas ciudades y barrios.

Mientras tanto, como bien señalan varios de las contribuciones que componen este monográfico, la actividad turística no se detuvo por completo durante mucho tiempo, si es que llegó a hacerlo. En cuanto se relajaron algunas de las medidas de confinamiento, e incluso antes, se pudo apreciar un movimiento importante de las ciudades hacia entornos imaginados como más rurales, bien como alternativa temporal de residencia, o bien como destino de descanso y distensión. Este fenómeno podría estar marcando el camino de nuevas tendencias hegemonizantes (Higgins-Desbiolles, 2020) y al mismo tiempo desplazando, de nuevo, los efectos socio-culturales del turismo para la construcción de masas desde espacios centrales (grandes urbes y destinos populares) hacia nuevas y viejas "periferias del placer" (Gaviria, 1977; Turner y Ash, [1975] 1991; Navarro-Jurado et al., 2015; Cañada y Murray, 
2019; entre otros) que de forma generalizada y desde finales de los sesenta del siglo pasado (de Kadt, 1979) ven en el turismo una oportunidad de crecimiento económico. Espacios en los que, como ya ha ocurrido en el pasado, los posibles conflictos resultan de menor interés informativo y son más difíciles de confrontar por parte de movimientos sociales locales (Gascón y Cañada, 2016).

\section{UNA DISYUNTIVA SISTÉMICA}

En su carrera por la incesante acumulación de capital, el capitalismo había reencontrado en el turismo una herramienta eficaz de extracción de plusvalías, ahora no sólo en zonas periféricas y semiperiféricas, sino también en el corazón de las capitales globales (Milano y Mansilla, 2019). De este modo, algunos efectos de las prácticas turísticas, largo tiempo conocidos, llegaron a lugares donde, muy poco tiempo antes, nadie los imaginaba y aparecieron otros nuevos frente a los que no tardaron en surgir movimientos de rechazo y protesta que señalan los riesgos del monocultivo turístico y de la toma de decisiones políticas orientadas hacia la satisfacción de las necesidades turísticas, a poner en valor y no a dar valor (Nogués-Pedregal, Travé-Molero, Carmona-Zubiri, 2017).

Podemos afirmar, por tanto, que la disyuntiva entre una huida hacia delante que profundice los rasgos más negativos del sistema -jerarquía, explotación, polarización [Wallerstein, [1991] 2007- o la articulación de una alternativa más igualitaria y redistributiva no se ciñe exclusivamente a la industria y las prácticas turísticas, ni apareció con la pandemia, sino que sobrevuela todo el entramado del sistema mundo capitalista desde el desfalco de 2007. No obstante, muchas de las primeras reacciones a los estragos de la COVID afirmaban que "saldríamos mejores" sin pararse a analizar si había datos fundados para semejante afirmación. Si como afirmara Immanuel Wallerstein, estamos ante una crisis estructural, en la que la incertidumbre a corto plazo se vuelve crónica y que nos sitúa ante dos posibles modelos, no debemos pasar por alto que uno de ellos cuenta con recursos y organización y el otro apenas con un aparato crítico desestructurado ${ }^{1}$.

Como discurso ideológico el turismo ha conseguido ocupar una posición preeminente en los imaginarios globales de consumo, al mismo tiempo que conservaba y expandía su imagen de "pasaporte al desarrollo" (De Kadt, 1979). Desde esa posición se velan las contradicciones que surgen de un análisis profundo de los efectos negativos de las prácticas turísticas y se ha desarmado, en buena medida, a quienes se oponen a su crecimiento sin límites o piden, al menos, no caer en el monocultivo, deslegitimados popularmente como turismófobos. Parafraseando la cita apócrifa de Fredric Jameson es más fácil imaginar un fin al capitalismo que un fin al turismo. En este sentido, el turismo post-COVID no será otra cosa (no podría serlo) que lo que sea la sociedad post-COVID. No es un hecho baladí, el carácter discursivo del turismo dificulta pensarlo como fenómeno social en toda su complejidad, velando las diversas prácticas turísticas que actúan e impactan (para bien y para mal) de muy diferentes formas en los territorios. Prácticas que podrían ser el germen de formas post-capitalistas de hacer turismo, más justas y mejor adaptadas a los acuciantes límites del planeta (Fletcher et al. 2021), o que pueden acabar siendo absorbidas e instrumentalizadas como estrategias de márquetin y creación de nueva demanda.

\footnotetext{
${ }^{1}$ En 2008, pensando en la anterior crisis, Wallerstein todavía era relativamente optimista: "Creo que, por desgracia, es igual de posible que se presencie la instalación de un sistema de explotación aún más violento que el capitalismo, como que se establezca un modelo más igualitario y redistributivo" (Reverchon, 2008)
} 
Si como sostiene Antonio Miguel Nogués-Pedregal "para cambiar el modelo de turismo, antes debemos cambiar la sociedad, nuestras prácticas sociales y, sobre todo, el uso que hacemos de nuestro tiempo libre" (Yanes y Travé-Molero, 2020: 194), la pregunta no debería ser cómo será el turismo después de la COVID-19, sino cómo será la sociedad. Hablar del futuro carece de sentido, pero los trabajos aquí publicados ya han detectado transformaciones importantes, estudiar su permanencia y analizar la orientación que acaben tomando será un trabajo para el que en los años venideros este monográfico será de gran ayuda.

\section{EL TURISMO POST COVID-19}

En un contexto académico en el que la discusión alrededor del futuro del turismo se ha convertido en una urgencia epistemológica (Schweinsberg et al., 2021), con este monográfico buscamos mejorar la comprensión de los nuevos movimientos y tendencias que en unos casos han surgido con y en otros han sido acelerados por la pandemia.

Los ocho primeros artículos que componen este número comparten la intención de documentar y analizar cambios socio-culturales detectados durante la pandemia y las posibilidades de permanencia a medio y largo plazo.

Pablo Díaz Rodríguez y Alberto Jonay Rodríguez Darias en Aprendiendo de la COVID en Canarias. El pesca-turismo como propuesta de diversificación económica en Tenerife, analizan las posibilidades que ofrece el modelo pesca-turismo como alternativa a la masificación del sol y playa y a la tendencia a la terciarización de las economías locales volcadas en la actividad turística. Los destinos más golpeados por la pandemia son, precisamente, los que con más urgencia deberían replantear su futuro tras la misma, siendo esta investigación un buen ejemplo de estrategias que permitirían aprovechar la experiencia turística para animar otros sectores económicos y la vitalidad social de contextos ya marcados por las consecuencias más negativos del turismo.

José Antonio Mansilla López y Neil Hughes con "En dos años no nos vamos a acordar de la pandemia”. Análisis del discurso sobre el decrecimiento turístico en Barcelona se acercan a los movimientos sociales que llevan años señalando los efectos negativos de la creciente popularidad turística de Barcelona sobre la calidad de vida de la población. Su trabajo muestra el desequilibrio entre el discurso hegemónico, avalado por la industria, que pide un regreso acelerado a la normalidad y el de los movimientos sociales que apuestan por el decrecimiento.

En Impacto socio-ecológico de la COVID-19 en el medio rural. Un caso de estudio en el centro de España, Cecilia Arnaiz-Schmitz, José María Rey-Matesanz, Cristina HerreroJauregui, Francisco Arques Soler y María F. Schmitz analizan cómo la COVID-19 ha modificado las condiciones socio-ecológicas de muchos municipios rurales y cómo esto, ejemplificado en los municipios rurales de la Comunidad de Madrid, puede suponer una oportunidad socio-económica de la mano de una migración urbano-rural y el aumento de su popularidad como lugares de visita y recreo identificados como más seguros.

Sílvia Margalef Callau con Masificación turística en espacios naturales: El caso del Parque Natural del Delta del Ebro en tiempos de COVID-19 presenta un estudio de caso que ejemplifica el aumento de visitas a espacios naturales de la mano del cambio de prácticas impulsado por la COVID. El caso del Parque Natural del Delta del Ebro sirve a la autora para señalar los impactos negativos que pueden aparecer ante el aumento descontrolado de las visitas turísticas y proponer alternativas de gestión que pudieran permitir formas de turismo social y ambientalmente más justas. 
El artículo de Ángel Iglesias Alonso La gobernanza local del turismo rural como respuesta a los efectos de la COVID-19 también señala el aumento de la popularidad de los destinos rurales y de cercanía para el turismo doméstico, en un estudio que recoge información de toda España. Este trabajo supone una contribución importante al desarrollo de la teoría sobre la gobernanza local en relación con la gestión turística y propone pautas de actuación para mejorar la toma de decisiones sobre desarrollo local a través del impulso al turismo rural.

Zuzana Kvítková, Zdenka Petrů y Alžběta Zíková en, Domestic tourism, its potential to compensate the outage of international arrivals caused by COVID-19 and the vulnerability of different groups of countries (a cluster analysis) ${ }^{2}$ analizan cuantitativamente la diferente capacidad que el turismo nacional puede tener para ayudar a recuperar la actividad de la industria de los viajes. Las autoras de esta investigación comparan datos de 41 países e identifican los factores decisivos para prever los posibles aumentos de los viajes turísticos domésticos y los resultados que, en función de estos aumentos, puede esperar cada destino.

José Miguel Moreno Carrillo y Diego Santos González presentan en El conflicto social y el COVID-19 en Latinoamérica y el Caribe: El sector turístico como daño colateral un análisis de los impactos sociales que la paralización de la actividad turística ha tenido sobre la población más vulnerable en esta parte del mundo. Los autores señalan la probable pervivencia de las medidas de higiene y seguridad que reducen la percepción del riesgo, así como la necesidad de apoyo público a la digitalización y modernización tecnológica del sector turístico en las zonas rurales latinoamericanas para permitirles tanto recuperarse del golpe propinado por la pandemia como aprovechar las oportunidades que, para estos territorios, comienza a ofrecer.

Um corpo que habita é capaz de (re)pensar hábitos: Reflexões para o turismo póspandemia de Newton Fernandes de Ávila y Maria Luiza Cardinale Batista, expone desde unos sólidos principios teóricos una investigación cualitativa que nos acerca a las vivencias, en muy diferentes planos, de quienes habitaron contextos turísticos en el sur de Brasil durante lo peor de la pandemia. El trabajo de Fernandes de Ávila y Cardinale Batista muestra el cuerpo como un lugar de vida que puede ayudar a pensar alternativas al futuro del turismo.

Los dos últimos artículos de este número monográfico transitan por un terreno más teórico, si bien comparten con los anteriores el objetivo de extraer enseñanzas útiles del proceso vivido.

Mariana De Oliveira Castanhas Casal Ribeiro con Reflection on COVID-19 pandemic and crisis management in tourism ${ }^{3}$ ofrece un análisis de cómo diferentes tipos de crisis han afectado y han sido enfrentadas por la industria turística, repasa los modelos de gestión de crisis en el turismo y propone el desarrollo de modelos de gestión específicos que puedan enfrentarse en el futuro a situaciones parecidas a la provocada por la COVID-19.

La sección de teoría y empiria acaba con el artículo de Rafael Cruces Portales Riesgos, vulnerabilidad y paradojas del turismo, un análisis desde la epistemología crítica del escenario provocado por la pandemia. El texto de Rafael Cruces resalta las paradojas del turismo y plantea la necesidad de repensar los modelos turísticos hegemónicos para enfrentar unas contradicciones que la pandemia ha hecho más visibles, pero que siempre habían estado ahí.

\footnotetext{
${ }^{2}$ El turismo nacional, su potencial para compensar la interrupción de las llegadas internacionales causada por la COVID-19 y la vulnerabilidad de diferentes grupos de países (un análisis de conglomerados)

${ }^{3}$ Reflexión sobre la pandemia de COVID-19 y la gestión de crisis en el turismo
} 
Cerramos este número especial con la sección de textos y glosas en la que se reseñan los libros "Covidosofía. Reflexiones filosóficas para el mundo postpandemia" (Paidós, 2020), coordinado por Dulcinea Tomás Cámara y "La España de las piscinas. Cómo el urbanismo neoliberal ha conquistado España y transformado su mapa político" (Arpa Editores, 2021) de Jorge Dioni López.

\section{CONCLUSIONES}

Cada uno de los artículos que componen este número tiene un valor científico en sí mismo $\mathrm{y}$ es un aporte, pequeño pero importante, al acervo del conocimiento que las Ciencias Sociales llevan años acumulando sobre el turismo.

No obstante, creemos que como conjunto este monográfico aporta una perspectiva completa sobre las diferentes disciplinas, con sus técnicas y sus metodologías, que desde las Ciencias Sociales se interesan por ese conjunto de prácticas sociales y procesos económicos y políticos que por comodidad textual y por acortar llamamos turismo, aportando, por tanto, una visión ampliada del fenómeno en su relación con la COVID-19. Además, como se ha señalado más arriba, los trabajos aquí presentados llegan de manera autónoma a resultados coincidentes en determinados ámbitos: crecimiento del turismo doméstico, valorización de los destinos imaginados como rurales y aparición de movimientos críticos en contextos masificados. Debemos tomar esto como indicador de la fiabilidad y relevancia de cada contribución por separado, pero especialmente del monográfico como un todo.

Esperamos que su lectura ilustre sobre los procesos que estamos viviendo y sirva para que futuras investigaciones dispongan de una base sólida sobre los orígenes de los mismos.

\section{BIBLIOGRAFÍA}

Butcher, J. (2020). "Response to journal of sustainable tourism article". En: https://politicsoftourism.blogspot.com/2020/08/response-to-journal-of-sustainable.html?m=1.

De Kadt, E. (Ed.) (1979). Tourism Passport to development. New York: Oxford University Press.

Ernest Cañada e Ivan Murray (eds.) Turistificación global. Barcelona: Icaria Editorial.

Fletcher, R., Blanco-Romero, A., Blázquez-Salom, M., Cañada, Murray Mas, I., Sekuloba, F. (2021). Caminos hacia un turismo post-capitalista. Informes en Contraste, núm. 18. Barcelona: Alba Sud.

Fotocasa (2021) "Casi el 40\% de los pisos turísticos se mantiene en el alquiler residencial tras un año de pandemia". En https://s36216.pcdn.co/wp-content/uploads/2021/08/NdP-alquilervacacional_2021.pdf [consulta 07/12/2021]

Gascón, J. y Cañada, E. (Coords), (2016) Turismo residencial y gentrificación rural. El Sauzal (Tenerife) \& Xixón: PASOS, RTPC \& Foro de Turismo Responsable.

Gaviria, M. (Coord.) (1974) España a go-go. Turismo chárter y neocolonialismo del espacio. Madrid: Ed. Turner.

Higgins-Desbiolles, F. (2020). “The 'war over tourism': Challenges to sustainable tourism in the tourism academy after COVID-19". Journal of Sustainable Tourism, 1-19.

INE (2020) "Medición del número de viviendas turísticas en España y su capacidad. Agosto de 2020". En https://www.ine.es/prensa/experimental viv turistica.pdf [consulta 07/12/2021]

Milano, C. y Mansilla, J. (Coords), (2019) Ciudad de vacaciones. Conflictos urbanos en espacios turísticos. Barcelona: Pol·len \& OACU.

Navarro-Jurado, E., Thiel-Ellul, D., \& Romero-Padilla, Y. R. (2015). "Periferias del placer: cuando turismo se convierte en desarrollismo inmobiliario-turístico". Boletín De La Asociación De Geógrafos Españoles, (67). https://doi.org/10.21138/bage.1826 
Nogués-Pedregal, A., Travé-Molero, R. \& Carmona-Zubiri, D. (2017) “Thinking against 'empty shells' in tourism development projects". EtnološkaTribina, 40(47): 88-108. https://doi.org/10.15378/1848-9540.2017.40.02

Organización Mundial del Turismo (2019) Panorama del turismo internacional, edición 2019, Madrid: OMT. https://doi.org/10.18111/9789284421237.

Organización Mundial del Turismo (2020) Barómetro OMT del Turismo Mundial mayo 2020. Madrid: OMT. https://doi.org/10.18111/9789284421831

Organización Mundial del Turismo (2021) Panorama del turismo internacional, Edición 2020, Madrid: OMT. https://doi.org/10.18111/9789284422746.

Reverchon A. (2008) "El capitalismo se acaba. Entrevista a Immanuel Wallerstein." https://rebelion.org/el-capitalismo-se-acaba/ [consulta 07/12/2021]

Schweinsberg, S., Fennell, D., \& Hassanli, N. (2021) "Academic dissent in a post COVID-19 world". Annals of Tourism Research, 91, 103289. https://doi.org/10.1016/j.annals.2021.103289

Turner, L. y Ash, J. (1991) La Horda Dorada. El turismo internacional y la periferia del placer. Madrid: Editorial Endymión.

Wallerstein I. (2007) Geopolitica y geocultura: ensayos sobre el moderno sistema mundial. Barcelona: Kairos

Yanes, S., Travé, R. (2020) “El turismo o la creación más perfecta y sofisticada del sistema de valores capitalista: Una entrevista a Antonio Miguel Nogués Pedregal". Perifèria, revista de recerca $i$ formació en antropologia, 25(3), 174-207. https://doi.org/10.5565/rev/periferia.819

\section{Breve currículo}

\section{Raúl Travé Molero}

Docente e investigador en el departamento de Ciencias Sociales y Comunicación de EAEOstelea-URJC, escuela universitaria de turismo y hospitalidad. Doctor en antropología social y cultural por la Universitas Miguel Hernández de Elche es, además, licenciado en periodismo por la Universidad Complutense de Madrid y en antropología social y cultural por la UMH. Ha sido investigador invitado en la Universidad de Ljubljana.

Sus trabajos de investigación han abordado los procesos de patrimonialización, comunicación y creación de hegemonía en contextos turísticos.

Es miembro de los grupos de investigación CULTURDES-UMH (Cultura, Turismo y [cooperación al] Desarrollo) e IDITUR-EAE-Ostelea (Centro de Investigación, Divulgación e Innovación Turística).

\section{Diego Santos González}

Diego Santos González (Pontevedra, 1984). Docente e investigador en el departamento de Ciencias Sociales y Comunicación de EAE-Ostelea-URJC, escuela universitaria de turismo y hospitalidad. Doctor en Sociología (Universidad Rey Juan Carlos, 2016), ha sido capaz de conjugar sus inquietudes intelectuales, sus pasiones personales y profesionales en una tesis doctoral titulada "El mundo sobre las olas. Perspectiva de desarrollo turístico a través del surfing".

Nacer en una familia de agricultores, artistas y deportistas, marcan su personalidad y sus inquietudes personales, por lo que el surf, los viajes, la escritura y la vida rural, son siempre el eje de sus relatos e investigaciones sociales.

Es miembro del grupos de investigación IDITUR-EAE-Ostelea (Centro de Investigación, Divulgación e Innovación Turística) 